\title{
Study of Ion Beam Shaping of an Anode-Type Ion Source Coupled with a Whenelt Mask
}

\author{
Yunsung Huh", ${ }^{\mathrm{a}, *}$ Yunseok Hwang ${ }^{\mathrm{a}}$, and Jeha $\mathrm{Kim}^{\mathrm{b}, *}$ \\ ${ }^{a} R \& D$ Center, Finesolution Co., Ltd., Suwon 16642, Korea \\ ${ }^{b}$ Department of Energy Convergence Engineering, Cheongju University, Cheongju 28503, Korea
}

Received June 29, 2018; revised July 14, 2018; accepted July 30, 2018

\begin{abstract}
We fabricated an anode-type ion source driven by a charge repulsion mechanism and investigated its beam shape controlled by a Whenelt mask integrated at the front face of the source. The ion beam shape was observed to vary by changing the geometry of the Whenelt mask. As the angle of inclination of the Whenelt mask was varied from $40^{\circ}$ to $60^{\circ}$, the etched area at a thin film was reduced from $20 \mathrm{~mm}$ to $7.5 \mathrm{~mm}$ at the working distance of $286 \mathrm{~mm}$, and the light transmittance through the etched surface was increased from $78 \%$ to $80 \%$, respectively. In addition, for the step height difference, $\Delta$ between the inner mask and the outer mask of $\Delta=0,-1 \mathrm{~mm}$, and $+1 \mathrm{~mm}$, we observed the ion beam shape was formed to be collimated, diverged, and focused, respectively. The focal length of the focused beam was $269 \mathrm{~mm}$. We approved experimentally a simple way of controlling the electric field of the ion beam by changing the geometry of the Whenelt mask such that the initial direction of the ion beam in the plasma region was manipulated effectively.
\end{abstract}

Keywords: Anode type ion beam source, Whenelt mask, Ion beam shape, Charge repulsion mechanism, Focused ion beam

\section{Introduction}

At present, micro-lenses that realize virtual reality and augmented reality are widely used in the information and communication technology (ICT) industry. In fabrication of the lenses the injection method using a jig is now popular in most processes. However, the control of the roughness of the surface of the jig and the ultrafine machining technology have become a subject of interest [1,2]. Wet [3] and dry [4] etching methods are known as ways for the planarization of the tool marks on the jig surface. In case of dry etching, anisotropic ion milling method using an ion beam is also widely used $[5,6]$.

In order to perform the ion milling process effectively, the shape of the ion beam must be changed according to the objective of the process. In case of etching a very small area, there is a demand for a way of forming intricately the ion beam reaching the area where the surface treatment is needed to be as small as several millimeters or less. There are ion millers developed for manufacturing transmission electron microscope (TEM) specimens, but they are unsuitable for industrial use because they can only etch very narrow area within a few micro-meters. Therefore, it is desired to develop a method of producing ion beam

*Corresponding author

E-mail:yhuh@finesolution.co.kr, jeha@cju.ac.kr shape that could be used to obtain a desired shape of ion beam within the working distance, such as a micro-lens jig or an industrial ion beam sputter [7].

Usually, electrons are relatively easy to deflect into a small radius by applying an electromagnetic field during the emission and acceleration process. In contrast, it is not simple to manipulate positive $(+)$ ions into a desired direction. It is the same phenomenon that the same charge that is not within the coherence length is accelerated and focused, which is not easy [8].

In this study, the anode-type ion source driven by the charge repulsion mechanism was fabricated, and the shape control and characteristics of the emitted ion beam according to the individual structure type of the Whenelt mask were investigated.

\section{Experiments}

In this study, the plasma complex machining center $\left(\mathrm{PCMC}^{\mathrm{TM}}\right.$, Finesolution Co.) was used to produce the beam shape of the ion source, and the conditions for the generation and acceleration of the plasma ion beam were controlled accordingly. The ion source was mounted in the PCMC as shown in Fig. 1.

The anode type ion source FPG-C100S (Finesolution Co., Korea), which is driven by the charge repulsion mechanism, oscillates the plasma using the gas existing in 


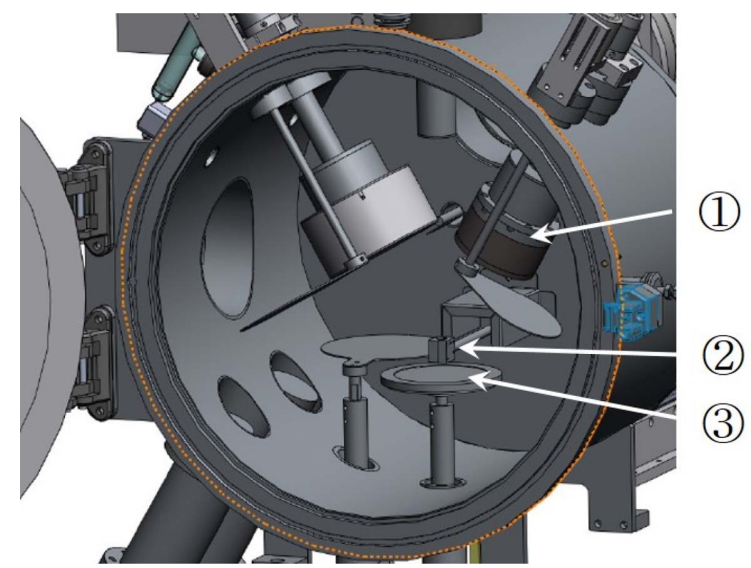

Figure 1. Configuration of the $\mathrm{PCMC}^{\mathrm{TM}}$ vacuum chamber with ion beam source; (1) charge repulsion type ion source, (2) Faraday sensor, (3) substrate holder.

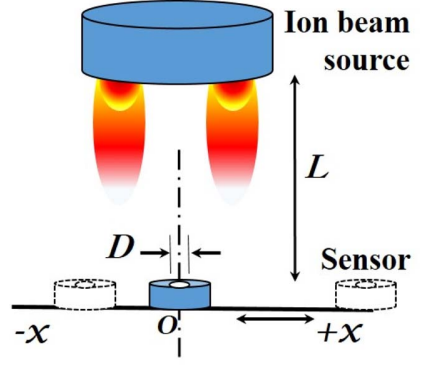

(a)

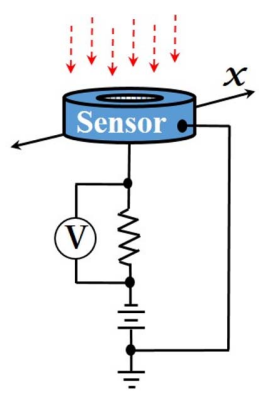

(b)
Figure 2. (a) Schematic of the ion current measuring device and (b) an equivalent circuit of the Faraday sensor.

the vacuum chamber without injecting the plasma generating gas into the ion source [9-11]. A voltage of 2,000 to $2,500 \mathrm{~V}$ was applied to the anode electrode to accelerate plasma generation and ions. High purity $(5 \mathrm{~N})$ argon was used as a gas of plasma generation, and the gas injection amount was adjusted in the range of 3 to $10 \mathrm{sccm}$ using mass flow controller (MFC). For normal beam emission, the gas pressure of the vacuum chamber was maintained at $2.8 \times 10^{-3}$ Torr, which is lower than the critical pressure [12], and was measured using a Pirani gauge, a full range gauge (Pfeiffer) and a capacitance diaphragm gauge.

We prepared $\mathrm{NiCr}$ thin films on glass substrate $(60 \times 60$ $\times 0.7 \mathrm{~mm}$ ) by sputtering that was used to study the shape of the ion beam as well as the light transmittance as measured with the diode array spectrometer (Hewlett Packard, 8452A). The initial transmittance of the thin film was $16 \%$ and the etch depth of the thin film was compared among specimens. The etching by the ion beam source was performed at the chamber pressure of $2 \mathrm{mTorr}$ for 90 seconds. For the analysis of the ion beam shape, we used a moving type Faraday sensor as shown in Fig. 2. The ion current was measured while the sensor was moving or stopping in the direction perpendicular to the ion beam.

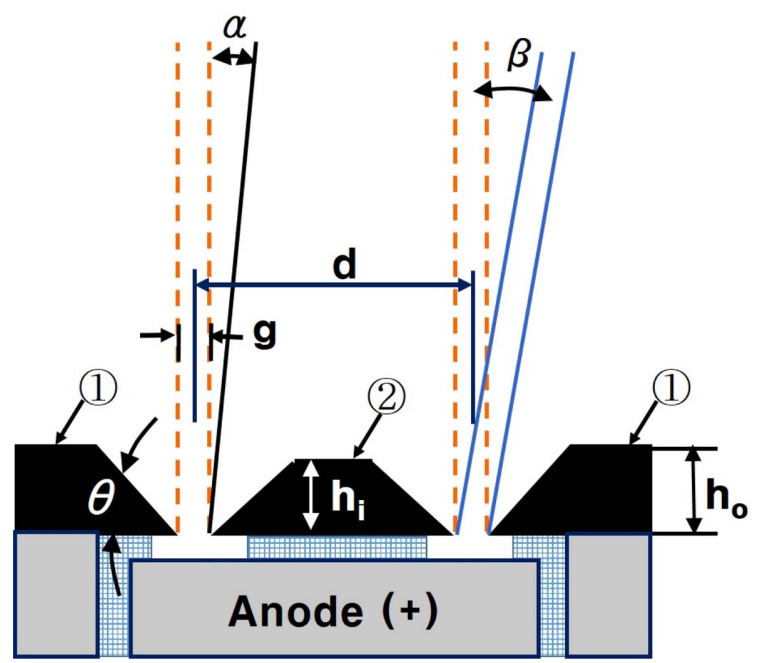

Figure 3. Cross sectional view of the Whenelt mask at the face of ion beam source and a dispersion form of the emission beam for the repulsion ion source: (1) outer mask, (2) inner mask.

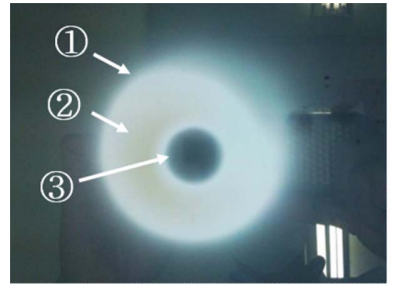

(a)

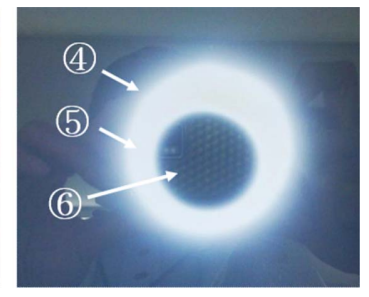

(b)
Figure 4. The shape of the $\mathrm{NiCr}$ thin film etched by the ion beam for the mask inclination angle (a) $40^{\circ}$, (b) $60^{\circ}$.

\section{Results and Discussion}

Figure 3 shows the cross-sectional structure of a set of Whenelt mask located at the front face of the anode-type ion source and the dispersion pattern of the emission beam. In Fig. 3, (1) and (2) represent the outer mask and the inner mask, respectively. In the figure, $\theta$ is the inclination angle of the mask, and $h$ is the mask height. $\alpha$ is a slit dispersion angle showing a difference based on the mask slit interval $g$, where $+\alpha$ and $-\alpha$ represent shapes in which beams emitted from one slit are dispersed or gathered, respectively. $\beta$ represents the dispersion angle of the whole beam showing whether the two beams are converged inwardly or dispersed outwardly based on the distance $d$ between the two slits and the slits formed by the inner mask and the outer mask. Here, $-\beta$ and $+\beta$ indicate the shape in which the two beams are gathered toward each other and the shape in which they are dispersed. As with the electron gun, the Whenelt mask plays a role of preventing the formation of a charge cloud due to the ions in the plasma and setting the initial motion direction in the case of the anode type ion source driven by the charge repulsion mechanism $[13,14]$. The shape of the beam according to the inclination angle $\theta$ and the height $h$ of the Whenelt mask shown in Fig. 3(a) was investigated using 


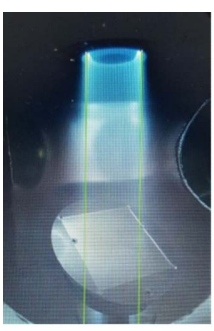

(a)

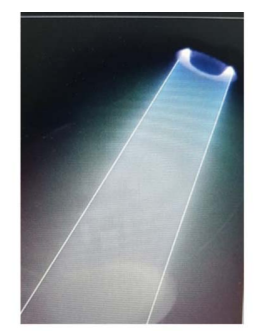

(b)

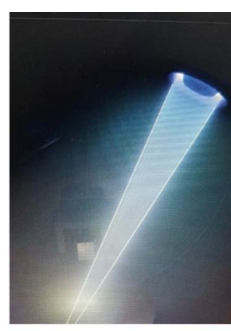

(c)
Figure 5. Emission ion beam shape at the inclination angle of mask $\theta=60^{\circ}$; (a) $\Delta=0$, (b) $\Delta=-1$, and (c) $\Delta=+1$.

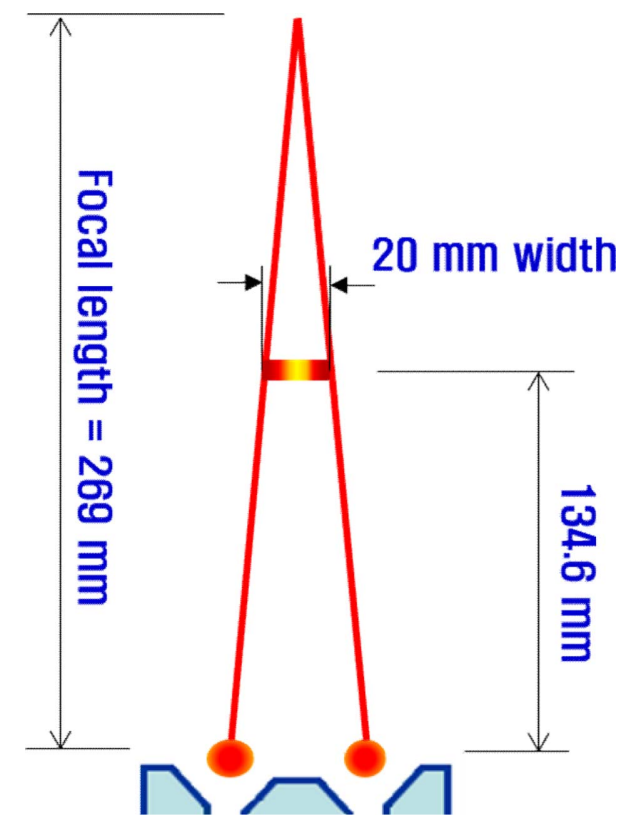

Figure 6. Emission of focused ion beam.

the thin film etching phenomenon by the ion beam.

Figure 4 shows the shape of the $\mathrm{NiCr}$ thin films etched by the ion beam. The working distance between the substrate and the ion beam source was $286 \mathrm{~mm}$ and etched for 90 seconds. The inclination angles $(\theta)$ of the Whenelt mask for (a) and (b) in Fig. 4 are $40^{\circ}$ and $60^{\circ}$, respectively. Since the shape of the beam ejected from the ion source is formed by the drift close loop movement of electrons, the etched shape is also donut shape similar to the ion source shape.

Figure 4(a) displays a donut shape with the inner and outer diameter of $20 \mathrm{~mm}$ and $60 \mathrm{~mm}$, and the transmittances for a wavelength of $\lambda=550 \mathrm{~nm}$ measured at the positions (1), (2), (3) were $31 \%, 78 \%$ and $30 \%$, respectively. Figure 4(b) shows the inner and outer diameters of the etched region are $30 \mathrm{~mm}$ and $45 \mathrm{~mm}$, and the light transmittances at $\lambda=550 \mathrm{~nm}$ measured at (4), (5), (6) were $23 \%, 80 \%$, and $19 \%$, respectively. The ion beam dispersion angles for the mask inclination angles $\theta=40^{\circ}$ and $60^{\circ}$ were measured $\alpha=+1.85^{\circ}$ and $+0.6^{\circ}$, respectively. The larger the mask inclination angle $\theta$, the smaller the dispersion angle $\alpha$ of the emitted beam. It implies that the
(4)

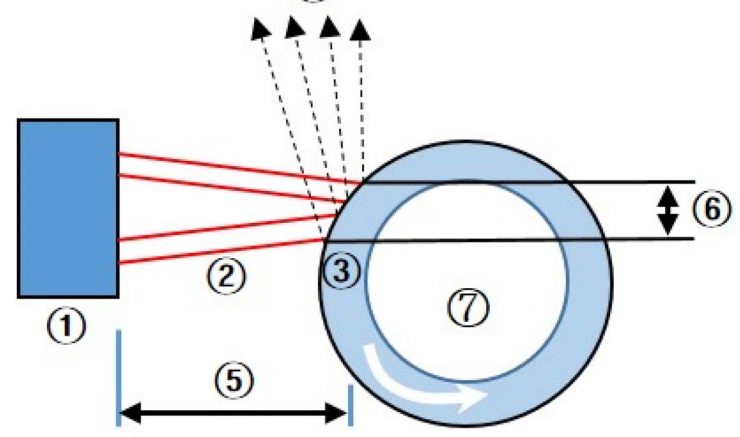

Figure 7. Schematic view of industrial ion beam sputtering concept (1) ion source, (2) emission beam, (3) cylindrical target, (4) sputtered material, (5) working distance, (6) beam sectional area, (7) cooling water.

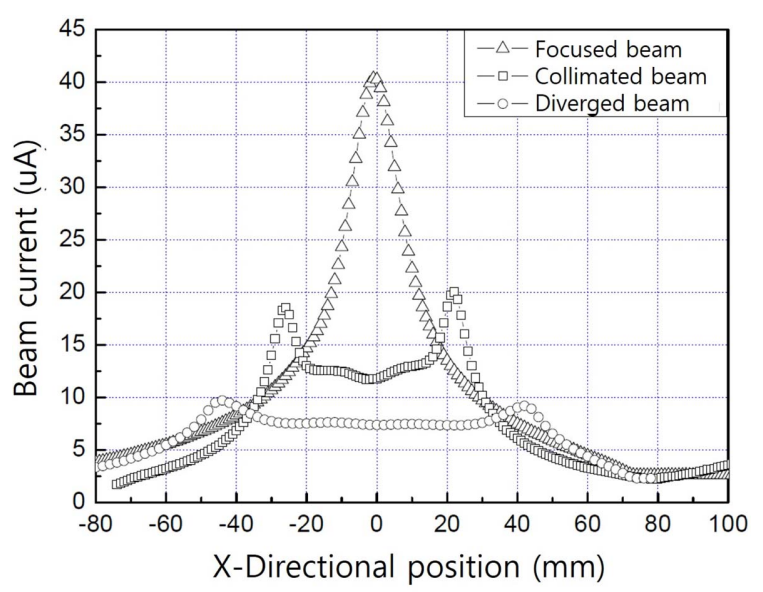

Figure 8. Ion currents measured along to the $x$-direction for collimated $(\square)$, diverged $(\bigcirc)$, and focused $(\triangle)$ beam shapes.

spreading of the accelerating ions becomes small and the ion density is increased, which results in a large etching effect.

The shape of the emission beam according to the height difference $\left(\Delta=h_{0}-h_{\mathrm{i}}\right)$ was investigated by changing the height $\left(h_{\mathrm{i}}\right)$ of the inner mask (Fig. 3 (2)) while maintaining the height $\left(h_{\mathrm{o}}\right)$ of the outer mask constant. For the normal beam emission, the gas pressure of the vacuum chamber was maintained at $2.8 \times 10^{-3}$ Torr, which was lower than the critical pressure $\left(P_{\text {crit }}=3.2 \times 10^{-3}\right.$ Torr $)$ [12].

Figure 5(a) shows the shape of the beam emitted when the inner and outer mask heights are equal to $5 \mathrm{~mm}$; that is, $\Delta=0$. The dispersion angle $\beta$ is $+0.45^{\circ}$, which is very similar to the result shown in Fig. 4(b), and shows a collimated beam shape close to a straight line. It is considered that the dispersion angle is developed due to the repulsion phenomenon caused by the same electric polarity during the acceleration of the emitted ion charge. Figure 5 (b) is a shape in which the height $\left(h_{\mathrm{i}}\right)$ of the inner mask is $1 \mathrm{~mm}$ higher than the outer mask height $\left(h_{0}\right)(\Delta=-1)$. A divergence beam of having an angle $\beta(\Delta=-1)=+7.5^{\circ}$ was formed. Figure 5(c) is a form in which the inner side 
is lower by $1 \mathrm{~mm}$ than the outer mask $(\Delta=+1)$. The shape of the emission beam becomes a focused beam having a dispersion angle $\beta(\Delta=+1)$ of about $-4.25^{\circ}$. As the height difference $\Delta$ was varied from -1 to +1 , the angle of dispersion $\beta$ was changed to $+7.5^{\circ}$ and $-4.25^{\circ}$, respectively. It is worth to note that there is an asymmetry in $\beta$, and its difference $\Delta \beta$ is as large as $11.75^{\circ}$. It is resulted from the repulsive force due to the same polarity of ions generated in the acceleration process.

For the focused ion beam shape as shown in Fig. 5(c), we estimated the focal length as in Fig. 6. The focused ion beam is formed in such a way that the ion beam emitted in a donut shape collects at one point. In the case of the focused ion beam of Fig. 5(c), the focal length is calculated as $269 \mathrm{~mm}$ when the slits distance $d=40 \mathrm{~mm}$ and the dispersion angle $b(\Delta=+1)=-4.25^{\circ}$. In practice, we suggest an example of the use of focused ion beam applied to the general industrial device as shown in Fig. 7. For $\beta$ $(\Delta=+1)=-4.25^{\circ}$, the focused ion beam was calculated to produce a cross section (6) of about $20 \mathrm{~mm}$ in diameter at the working distance (5) of nearly $135 \mathrm{~mm}$. The working distance (5) and the beam cross-section (6) are within typical values applicable to industrial use. Therefore, the focused ion beam source can be combined with a typical cylindrical sputter cathode target (3)), diameter $165 \mathrm{~mm}$ ) used in industry, and it could be associated with an ion beam sputtering apparatus.

We measured the ion current of beam for the cases of ion beam shapes shown in Fig. 5. Figure 8 displays the measured current along the $\mathrm{x}$-direction using the moving type Faraday sensor. For the experiment, argon of $10 \mathrm{sccm}$ was injected into the vacuum chamber, and the gas pressure $(P)$ of the vacuum chamber was 1.5 mTorr lower than the critical pressure $\left(P_{\text {crit }}\right)$. The voltage applied to the anode electrode was $1.5 \mathrm{kV}$, and the Faraday sensor for current measurement was moved in the $\mathrm{x}$-direction at a distance $L=185 \mathrm{~mm}$ away from the ion source. As shown in Fig. 8, the maximum current values of 19.5, 10, and $40 \mu \mathrm{A}$ were measured for the collimation, divergence, and focused beams, respectively. It can be confirmed that the beam current is significantly increased as the beam is collected at the central portion as in the focused type. This result is suggestive that the focused type ion beam would be very effective for a process requiring a high etch rate at a local position.

The phenomenon that has been shown through this study can be interpreted as follows. In the case of the anode electrode located at the lower part of the Whenelt mask and the positive voltage is applied, the relative potential corresponds to the ground potential around the anode electrode including the specimen support, the vacuum container and so on. The ions in the plasma are subjected to an electric force due to the electric field between the anode and the ground potential. If the pressure $(P)$ inside the vacuum chamber is above the critical pressure $\left(P_{\text {crit }}\right)$, a glow discharge is formed. But if the chamber pressure $(\mathrm{P})$ is lower than the critical pressure $\left(P_{\text {crit }}\right)$, a dark discharge is formed in which the mean free path of the gas molecules is sufficiently long. Therefore, the ions inside the plasma are accelerated toward the opposite potential facing the anode electrode without being influenced by the surrounding mask near the dark space region $[12,15]$. Thus, the shape of the Whenelt mask becomes very important to control the beam shape. It is because the main factor determining the initial acceleration direction for the plasma inner ions in the dark discharge state is the electric field.

From the results of this experiment, we confirmed that the shape of the ion beam varies depending on the inclination angle and the relative height between the inner and outer mask.

1) As the angle of inclination $(\theta)$ of the mask becomes small, the ion beam spreads widely and disperse that results in reduced light transmission for the etched $\mathrm{NiCr}$ film.

2) The relative height of the inner and outer sides of the Whenelt mask is very critical to control the whole beam shape in ways of inward or outward the center of the beam acceleration direction. When the inner mask is protruded 1 $\mathrm{mm}$ higher than the outer mask $(\Delta=-1)$, a divergent ion beam with a dispersion angle $b$ of $+7.5^{\circ}$ is emitted. On the other hand, when the inner mask is lowered by $1 \mathrm{~mm}$ $(\Delta=+1)$ a focused ion beam having a dispersion angle $\beta$ of $-4.25^{\circ}$ was emitted.

\section{Summary}

We investigated the shape of the ion beam depending on the shape of the Whenelt mask of the anode type ion source driven by the charge repulsion mechanism. The shape of the ion beam and the ion density were found to vary depending on the inclination angle $(\theta)$ and the height $(\Delta)$ of the Whenelt mask. It was confirmed that as the inclination angle of the mask increases, and as the height of the inner mask decreases $(\Delta>0)$, the ions emitted are being focused. In addition, we found that the focused ion beam can satisfy the working distance applicable to the ion beam sputtering apparatus and the beam sectional area. In this experiment, an ion beam with a focal length of $269 \mathrm{~mm}$ was formed at $\theta=60^{\circ}$ and $\Delta=-1$. It is conclusive that the electric field subjected to the ion source can be controlled by the geometrical shape of the Whenelt mask and the initial direction of the ion in the plasma region.

\section{References}

[1] D. S. Choi, T. J. Je, Y. E. Yoo, and E. S. Lee, Mon. Mecha. Eng. 34, 24 (2007)

[2] D. S. Choi, Y. E. Yoo, T. J. Je, and J. S. Yun, J. KSME 48, 52 (2008).

[3] J.-W. Heo, Y.-C. Jeon, C.-M. Jeong, and C.-S. Lim, J. Korean Acad. Prosthodont. 38, 178 (2000). 
[4] M. Grunze, H. Ruppender, and O. Elshazly J. Vac. Sci. Technol. A6, 1266 (1988)

[5] W. J. Park, A Study on customized plasma dry etching suited to various application processes, Ph.D. Thesis, Seoul Nat. Univ. (2012).

[6] J. J. Cuomo, S. M. Rossnagel, H. R. Kaufman, Handbook of Ion Beam Processing Technology: Principles, Deposition, Film Modification and Synthesis, (William Andrew Publishing, 1989), pp. 219-239.

[7] KAIST, Patent No. KR10-0975851, Aug. 9 (2010).

[8] K. S. Min, S. S. Lee, J. Y. Yoon, C.-W. Chung, E. S. Oh, Y. Hwang, and J.-T. Kim, Proceedings of the 48th Korean Vacuum Society Conference, pp. 145-1 (2014).
[9] Finesolution, Patent No. KR10-1478216, Dec. 24 (2014).

[10] Finesolution, Patent No. US9,269,535 B1, Feb. 23 (2016).

[11] Y. Huh, Y. Hwang, and J. Kim, Appl. Sci. Conv. Technol. 27, 47 (2018).

[12] Y. Huh, Y. Hwang, and J. Kim, submitted to Appl. Sci. Conv. Technol., June 8 (2018).

[13] R. G. Wilson and G. R. Brewer, Ion Beams With Applications to Ion Implantation, (John Wiley \& Sons, New York, 1973).

[14] N. Oudini, G. J. M. Hagelaar, J.-P. Boeuf, and L. Garrrigues, J. Appl. Phys. 109, 073310 (2011).

[15] D. Depla1, S. Mahieu1, and J. E. Greene, Sputter Deposition Processes, (William Andrew Publishing, 2010), pp. 261-265. 